\title{
On quantum and approximate privacy
}

\author{
Hartmut Klauck * \\ School of Mathematics \\ Institute for Advanced Study \\ Princeton, NJ08540, USA \\ klauck@ias.edu
}

\begin{abstract}
This paper studies privacy and secure function evaluation in communication complexity. The focus is on quantum versions of the model and on protocols with only approximate privacy against honest players. We show that the privacy loss (the minimum divulged information) in computing a function can be decreased exponentially by using quantum protocols, while the class of privately computable functions (i.e., those with privacy loss 0) is not enlarged by quantum protocols. Quantum communication combined with small information leakage on the other hand makes certain functions computable (almost) privately which are not computable using either quantum communication without leakage or classical communication with leakage. We also give an example of an exponential reduction of the communication complexity of a function by allowing a privacy loss of $o(1)$ instead of privacy loss 0 .
\end{abstract}

\section{Introduction}

Mafiosi $\mathrm{Al}$ and $\mathrm{Bob}$, both honest men, claim rights to protect a subset of the citizens of their hometown. To find out about possible collisions of interest they decide to communicate and find out whether there is a citizen they both intend to protect. Of course they would like to do this in a way that gives each other as little information as possible on the subset they think of. In other words, they want to compute a function with as much privacy as possible, rather than caring about the communication cost inclined. This problem is one of the kind studied in the theory of private computation resp. secure function evaluation, initiated by Yao 32. Another example is the two millionaires' problem, in which $\mathrm{Al}$ and Bob try to determine who is richer, but without revealing more about their actual wealth.

\footnotetext{
* Supported by NSF grant CCR-9987845. Work mostly done while at CWI and supported by the EU 5th framework program QAIP IST-1999-11234 and by NWO grant 612.055.001.
} 
Informally a protocol for the computation of some function on inputs distributed to several players is private, if all information that can be deduced by one player during a run of the protocol can also be deduced from the player's input and the function value alone. A function is private, if it can be computed by a private protocol. Generalizing this a function is said to have privacy loss $k$, if the minimum information divulged to the other players is $k$ in any protocol computing the function. In this definition we use an information theoretic measure for the privacy loss. Alternatively the information leakage of a protocol may be measured as a distance between message states that must be "almost indistinguishable" for a player. This setup generalizes several cryptographic scenarios, see [32].

There are some variants and twists to this model. One can distinguish computationally secure and information theoretically secure protocols. The first variant is studied e.g. in [32] and [18. Multiparty protocols in the information theoretically secure setting are given in [7] and [12]. A second kind of variations concerns the type of players. Basically "honest but curious" and "malicious" (or "byzantine") players have been considered in the literature. The first type of players sticks to the protocol but tries to get information by running some extra program on the messages received. The second type of players deviates arbitrarily from the protocol to get as much information as possible. Furthermore protocols may be deterministic, randomized, or use the possibilities offered by quantum communication. A quantum variant of (information-theoretically) secure multiparty computation with malicious players has been investigated recently in [17. A thorough study of secure quantum computation with honest players seems to be missing, especially in the two-player case. Lo has investigated the case of one-sided secure quantum computation [26, in which only one player learns the function value. Certain aspects of general two-party secure quantum computation are discussed in [27].

To compare the possible combinations of the above choices concerning the underlying model consider the following facts known in the non-quantum setting. The two millionaires' problem has a computational solution 32] relying on the existence of one-way functions, but it cannot be solved in the information theoretic sense [14] (not even among honest players), i.e., some cryptographic hardness assumption has to be used. A variant of the millionaires' problem that can actually be solved with information theoretic privacy for honest players is the identified minimum problem, in which the wealth of the less rich player and his identity is revealed, but no additional information about the wealth of the other player 23. Secure function evaluation among two dishonest players without computational restrictions is usually impossible. Information-theoretically secure multiparty protocols $(\geq 3$ players) with more than two thirds of all players being honest are possible for all functions [7], in the computationally secure setting it is possible to compute all functions when more than one half of all players are honest [18. 
In this paper we concentrate on information theoretical security and honest players. While considering only honest players seems to strongly restrict the model, it is important for several reasons. First, understanding honest players is a prerequisite to understanding actively cheating players. Secondly, these players capture "passive" attacks that cannot be detected in any way, which might be an important motivation for curious players to follow such a strategy. Furthermore [18] gives a quite general reduction from multiparty protocols with honest majority to protocols with only honest players (in the computationally secure setting). Other motivations for considering this model include close connections to complexity measures like circuit size 25]. Privacy loss may also be viewed as a complexity measure, having some useful connections to communication complexity exploited e.g. in [1], 3].

We focus on the following aspects of private computing. Al and Bob have heard that quantum computers can break cryptographic schemes classically assumed to be secure, so they do not want to rely on computational solutions $^{1}$. They are interested in whether quantum communication enlarges the set of privately computable functions or substantially decreases the privacy loss of functions. Furthermore they are interested in whether it is possible to decrease the communication cost of a protocol by allowing leakage of a small amount of information. We concentrate on the two player model in this paper, though some of the results have implications for the multiparty setting, which we mention in the conclusions.

The functions we mainly consider in this paper are the disjointness problem $D I S J_{n}$, in which $\mathrm{Al}$ and Bob each receive a subset of a size $n$ universe, and have to decide whether their subsets are disjoint or not, and the identified minimum problem $I d M i n_{n}$, in which $\mathrm{Al}$ and Bob receive numbers $x, y$ from 0 to $2^{n}-1$, and the output is $2 x+1$, if $x \leq y$, and $2 y$ otherwise.

The type of players we investigate are honest but curious. This means they stick to the protocol, but otherwise try anything they can to get information about the other player's input. In the quantum case a major point will also be whether the players might be trusted to not quit the protocol before the end. Our main model will measure the maximum information obtainable over all the rounds, not only the information obtainable at the end of the protocol. This corresponds to players that might quit the protocol before its end ${ }^{2}$. The other model of nonpreemptive players will be investigated also, but here every function turns out to be computable almost privately and at the same time efficiently in the quantum case.

Our main results are the following. We show that the quantum protocol for disjointness with communication complexity $O(\sqrt{n} \log n)$ given in [9] can be adapted to have privacy $\operatorname{loss} O\left(\log ^{2} n\right)$. We proceed to show that any

\footnotetext{
${ }^{1}$ Actually it is quite possible that quantum one-way permutations exist, see e.g. 6].

${ }^{2} \mathrm{Al}$ has the habit of shooting his guests after dessert, which may be well before the end of the protocol.
} 
classical bounded error protocol for disjointness divulges $\Omega(\sqrt{n} / \log n)$ bits of information. Thus $\mathrm{Al}$ and Bob are highly motivated to use the quantum protocol for privacy reasons. Note also that any (even nonprivate) classical randomized protocol for disjointness needs communication $\Omega(n)$ [20]. Every (nonprivate) quantum protocol for disjointness needs communication $\Omega(\sqrt{n})$, as recently established in 30 .

We then show that the class of privately computable functions is not enlarged by using quantum computers, i.e., every function that can be computed privately using a quantum protocol can also be computed privately by a deterministic protocol. This result leads to the same characterization of privately computable functions as in the classical case. We furthermore show that allowing a small leakage combined with quantum communication allows to compute Boolean functions which are nonprivate. This does not hold for both quantum communication without leakage and classical communication with leakage. We also analyze a tradeoff between the number of communication rounds and the leakage required to quantum compute a nonprivate function.

We then turn to the question, whether leakage can decrease the communication complexity and show that $I_{d M i n_{n}}$ can be computed with leakage $1 / \operatorname{poly}(n)$ and communication poly $(n)$, while any perfectly private (quantum) protocol needs $\Omega\left(2^{n}\right)$ rounds and communication. Thus a tiny leakage reduces the communication cost exponentially. It has been known previously [4] that one bit of privacy loss in the "hint sense" can decrease the communication complexity exponentially, but in our result the privacy loss is much smaller, and the function we consider is more natural than the example in 4.

The paper is organized as follows. In the next section we give the necessary definitions and some technical results. Section 3 describes the result about an exponential decrease of privacy loss by using quantum communication. Section 4 discusses the set of functions computable by private or almost private quantum protocols. Section 5 shows how allowing very small privacy loss can decrease communication complexity. In section 6 we give conclusions and some open problems.

\section{Preliminaries}

In this section we first describe the communication model we study, then the (quantum) information theoretic notions used, and finally discuss privacy definitions. For introduction to quantum computing see e.g. [29].

\subsection{The communication complexity model}

In the quantum communication complexity model 33, two parties $\mathrm{Al}$ and Bob hold qubits. When the game starts Al holds a superposition $|x\rangle$ and 
Bob holds $|y\rangle$ (representing the input to the two players), and so the initial joint state is simply $|x\rangle \otimes|y\rangle$. Furthermore each player has an arbitrarily large supply of private qubits in some fixed basis state. The two parties then play in rounds. Suppose it is Al's turn to play. Al can do an arbitrary unitary transformation on his qubits and then send one or more qubits to Bob. Sending qubits does not change the overall superposition, but rather changes the ownership of the qubits, allowing Bob to apply his next unitary transformation on the newly received qubits. Al may also (partially) measure his qubits during his turn. At the end of the protocol, one player makes a measurement and sends the result of the protocol to the other player. The overall number of message exchanges is called the number of rounds. In a classical probabilistic protocol the players may only exchange messages composed of classical bits.

The complexity of a quantum (or classical) protocol is the number of qubits (respectively, bits) exchanged between the two players in the worst case. We say a protocol computes a function $f: \mathcal{X} \times \mathcal{Y} \mapsto \mathcal{Z}$ with $\epsilon \geq 0$ error if, for any input $x \in \mathcal{X}, y \in \mathcal{Y}$, the probability that the two players compute $f(x, y)$ is at least $1-\epsilon$.

Sometimes we want to relax the above correctness requirement. We say a protocol $\mathcal{P}$ computes $f$ with $\epsilon$ error with respect to a distribution $\mu$ on $\mathcal{X} \times \mathcal{Y}$, if

$$
\operatorname{Prob}_{(x, y) \in \mu, \mathcal{P}}(\mathcal{P}(x, y)=f(x, y)) \geq 1-\epsilon .
$$

A randomized classical or a quantum protocol has access to a public coin, if the players can flip a classical coin and both read the result without communication. If not mentioned otherwise we do not consider this variant of the model.

The communication matrix of a function $f(x, y)$ is the matrix with rows labelled by the $x$ 's, columns labelled by the $y$ 's, and containing $f(x, y)$ at position $x, y$.

A rectangle in the communication matrix is a submatrix indexed by the product of a subset of the row-labels and a subset of the column-labels. A rectangle is monochromatic, if all its entries are the same.

\subsection{Information theory background}

The quantum mechanical analogue of a random variable is a probability distribution over superpositions, also called a mixed state. For the mixed state $X=\left\{p_{i},\left|\phi_{i}\right\rangle\right\}$, where $\left|\phi_{i}\right\rangle$ has probability $p_{i}$, the density matrix is defined as $\rho_{X}=\sum_{i} p_{i}\left|\phi_{i}\right\rangle\left\langle\phi_{i}\right|$. Density matrices are Hermitian, positive semidefinite, and have trace 1 . I.e., a density matrix has only real eigenvalues between zero and one, and they sum up to one.

The trace norm of a matrix $A$ is defined as $\|A\|_{\mathrm{t}}=\operatorname{Tr} \sqrt{A^{\dagger} A}$, which is equal to the sum of the magnitudes of the singular values of $A$. Note that 
if $\rho$ is a density matrix, then it has trace norm one. If $\phi_{1}, \phi_{2}$ are pure states then:

$$
\|\left|\phi_{1}\right\rangle\left\langle\phi_{1}|-| \phi_{2}\right\rangle\left\langle\phi_{2}\right| \|_{\mathrm{t}}=2 \sqrt{1-\left|\left\langle\phi_{1} \mid \phi_{2}\right\rangle\right|^{2}}
$$

The following important fact (the Kraus representation theorem) characterizes the physically allowed quantum operations on density matrices (trace preserving completely positive superoperators) in terms of adding blank qubits, doing unitary transformations, and tracing out (see [29]). Hence we can restrict our considerations to these operations.

Fact 1 The following statements are equivalent:

1. An operation $T$ sending density matrices over $H_{1}$ to density matrices over $\mathrm{H}_{2}$ is physically allowed (i.e., trace preserving and completely positive).

2. There is a Hilbert space $H_{3}$ with $\operatorname{dim}\left(H_{3}\right) \leq \operatorname{dim}\left(H_{1}\right)$ and a unitary transformation $U$, such that for all density matrices $\rho$ over $H_{1}$ the result of $T$ applied to $\rho$ is

$$
\text { trace }_{H_{1} \otimes H_{3}}\left[U\left(\rho \otimes\left|0_{H_{3} \otimes H_{2}}\right\rangle\left\langle 0_{H_{3} \otimes H_{2}}\right|\right) U^{\dagger}\right] .
$$

So allowed operations can be simulated by adding some blank qubits, applying a unitary transformation and "dropping" some qubits.

Another useful theorem states that for two mixed states $\rho_{1}, \rho_{2}$ their distinguishability is reflected in $\left\|\rho_{1}-\rho_{2}\right\|_{\mathrm{t}}[1]$ :

Fact 2 Let $\rho_{1}, \rho_{2}$ be two density matrices on the same space $\mathcal{H}$. Then for any measurement $\mathcal{O}$,

$$
\left\|\rho_{1}^{\mathcal{O}}-\rho_{2}^{\mathcal{O}}\right\|_{1} \leq\left\|\rho_{1}-\rho_{2}\right\|_{\mathrm{t}}
$$

where $\rho^{\mathcal{O}}$ denotes the classical distribution on outcomes resulting from the measurement of $\rho$, and $\|\cdot\|_{1}$ is the $\ell_{1}$ norm. Furthermore, there is a measurement $\mathcal{O}$, for which the above is an equality.

The Shannon entropy $H(X)$ of a classical random variable $X$ and mutual information $I(X: Y)$ of a pair of random variables $X, Y$ are defined as usual (see e.g. [16]).

The von Neumann entropy $S(\rho)$ of a density matrix $\rho$ is defined as $S(\rho)=$ $-\operatorname{Tr} \rho \log \rho=-\sum_{i} \lambda_{i} \log \lambda_{i}$, where $\left\{\lambda_{i}\right\}$ is the multi-set of all the eigenvalues of $\rho$. Notice that the eigenvalues of a density matrix form a probability distribution. For properties of this function see [29].

We use the following fact about the continuity of entropy (see theorem 16.3.2 in [16] and theorem 11.6 in [29]). 
Fact 3 Let $p, q$ be distributions on $\{0,1\}^{n}$ with $d=\|p-q\|_{1}=\sum_{x}\left|p_{x}-q_{x}\right| \leq$ $1 / 2$. Then

$$
|H(p)-H(q)| \leq d \cdot n-d \log d .
$$

Let $\rho, \sigma$ be states in a $2^{n}$ dimensional Hilbert space with $d=\|\rho-\sigma\|_{\mathrm{t}} \leq$ $1 / e$. Then

$$
|S(\rho)-S(\sigma)| \leq d \cdot n-d \log d .
$$

An immediate corollary of Jensen's inequality is the following:

Fact 4 For $x \in\{0,1\}^{n}$ let $0 \leq p_{x}$ and $\sum_{x} p_{x} \leq \gamma \leq 1$. Then

$$
-\sum_{x} p_{x} \log p_{x} \leq \gamma n-\gamma \log \gamma
$$

For a bipartite quantum state $\rho_{X Y}$ we define the "mutual information" $I(X: Y)$ as $I(X: Y)=S(X)+S(Y)-S(X Y)=S\left(\rho_{X}\right)+S\left(\rho_{Y}\right)-$ $S\left(\rho_{X Y}\right)$, where $\rho_{X}, \rho_{Y}$ are the reduced density matrices on the systems $X, Y$. We also define conditional mutual information $I(X: Y \mid Z)$ as follows:

$$
I(X: Y \mid Z)=S(X Z)+S(Y Z)-S(Z)-S(X Y Z) .
$$

We will employ the following facts from 22 .

Fact 5 (Average encoding theorem) Let $x \mapsto \rho_{x}$ be a quantum encoding mapping an $m$ bit string $x \in\{0,1\}^{m}$ into a mixed state with density matrix $\rho_{x}$. Let $X$ be distributed over $\{0,1\}^{m}$, where $x \in\{0,1\}^{m}$ has probability $p_{x}$, let $Q$ be the register holding the encoding of $X$ according to this map, and let $\bar{\rho}=\sum_{x} p_{x} \rho_{x}$. Then,

$$
\sum_{x} p_{x}\left\|\bar{\rho}-\rho_{x}\right\|_{\mathrm{t}} \leq \sqrt{(2 \ln 2) I(Q: X)} .
$$

A purification of a mixed state with density matrix $\rho$ over some Hilbert space $\mathcal{H}$ is any pure state $|\phi\rangle$ over some space $\mathcal{H} \otimes \mathcal{K}$ such that $\operatorname{trace}_{\mathcal{K}}|\phi\rangle\langle\phi|=$ $\rho$.

Fact 6 (Local transition theorem) Let $\rho_{1}, \rho_{2}$ be two mixed states with support in a Hilbert space $\mathcal{H}, \mathcal{K}$ any Hilbert space of dimension at least $\operatorname{dim}(\mathcal{H})$, and $\left|\phi_{i}\right\rangle$ any purifications of the $\rho_{i}$ in $\mathcal{H} \otimes \mathcal{K}$. Then, there is a local unitary transformation $U$ on $\mathcal{K}$ that maps $\left|\phi_{2}\right\rangle$ to $\left|\phi_{2}^{\prime}\right\rangle=I \otimes U\left|\phi_{2}\right\rangle$ such that

$$
\|\left|\phi_{1}\right\rangle\left\langle\phi_{1}|-| \phi_{2}^{\prime}\right\rangle\left\langle\phi_{2}^{\prime}\right|\left\|_{\mathrm{t}} \leq 2\right\| \rho_{1}-\rho_{2} \|_{\mathrm{t}}^{\frac{1}{2}}
$$




\subsection{Privacy}

Given a protocol a player is honest if for each input and all messages he receives he sends exactly the messages prescribed by the protocol. All operations are allowed as long as this requirement is met. It is e.g. allowed to copy a classical message to some additional storage (if it is known that the message is classical). Copying general unknown quantum states is, however, impossible 31.

We state this requirement a bit more formal in the following way. In a quantum protocol as defined above the actions of the players are defined as a series of unitary transformations plus the sending of a certain choice of qubits. For player $\mathrm{Al}$ to be honest we demand that for all rounds $t$ of the protocol, for all inputs $x$, and for all sequences of pure state messages he may have received in the previous rounds, the density matrix of the message in the next round equals the density matrix defined by the protocol and the input. Note that in a run of the protocol the player might actually receive mixed state messages, but the behavior of the player on these is defined by his behavior on pure state messages.

We define the privacy loss of a protocol as follows. Let $\rho_{A B X Y}$ denote a state of the registers containing Al's private qubits in $A$, Bob's private qubits in $B$, Al's input in $X$, Bob's input in $Y$. We assume that the (classical) inputs are never erased by the players.

For a distribution $\mu$ on the inputs to a protocol computing $f$ the information divulged to Bob at time $t$ is $L(t, B, \mu)=I(B: X \mid Y, f(X, Y))$, for the state $\rho_{A B X Y}^{(t)}$ of the protocol at time $t$ induced by the distribution $\mu$ on the inputs. Symmetrically we define Al's loss $L(t, A, \mu)$. The privacy loss of a protocol is the supremum of $L(t, \cdot, \mu)$ over all $t$ and $A, B$ and all $\mu$.

The privacy loss $L_{\epsilon}(f)$ of a function $f$ is the infimum privacy loss over all quantum protocols computing $f$ with error $\epsilon$. The classical privacy loss $C L_{\epsilon}(f)$ is defined analogously, with the infimum over all classical randomized protocols.

A function $f$ is said to be private, if $C L_{0}(f)=0$. It is known that $C L_{\epsilon}(f)=0$ with $\epsilon<1 / 2$ holds only for private functions [23].

Note that in the above definition we have assumed that the information available to a player is small in all rounds. Thus even if one player decides to quit the protocol at some point the privacy loss is guaranteed.

If we consider only the final state of the protocol in our definition we call the players honest and nonpreemptive. For a classical protocol there is no difference between these two possibilities, since the information available only increases with time. In the quantum case, however, this is not true.

The information divulged by a nonprivate protocol can also be measured in a different way, namely via distinguishability, see [14]. Let $\rho_{A B}^{x y}$ denote the state of Al's and Bob's qubits in some round for inputs $x, y$, and let

$\rho_{A}^{x y}$ resp. $\rho_{B}^{x y}$ denote the reduced density matrices on Al's and Bob's qubits. 
A protocol is said to leak at most $\delta$ to Bob, if for all $x, x^{\prime}$ and $y$ with $f(x, y)=f\left(x^{\prime}, y\right)$ it is true that

$$
\left\|\rho_{B}^{x y}-\rho_{B}^{x^{\prime} y}\right\|_{\mathrm{t}} \leq \delta
$$

This means that no quantum operation on Bob's qubits can distinguish the two states better than $\delta$. Thus there is a limit on Bob's ability to distinguish Al's inputs as long as changing these does not change the output. An analogous definition is made for Al. We say a protocol leaks at most $\delta$, if the maximum leakage to a player in any round is at most $\delta$.

Two more definitions of (classical) privacy loss are considered in [4. In the first variant there is not one protocol which is good against all distributions on the inputs, but for each distribution there may be one specialized protocol, for which the privacy loss is measured. The second definition is privacy loss in the hint sense, here a function $h$ is computed privately instead of a function $f$, and $f(x, y)$ can be computed from $h(x, y)$. The privacy loss is the difference between the logs of the ranges of $f$ and $h$. We generally use privacy loss with respect to the definition we have given first.

Let us show that our standard definition of privacy loss and the first alternative definition mentioned above are asymptotically equivalent for randomized and quantum protocols. The following lemma is a consequence of the standard Yao principle (von Neumann duality), see [24].

Lemma 7 The following statements are equivalent in the sense that if one is true for some values $\epsilon, \delta$, then the other is true with values $2 \epsilon, 2 \delta$.

- There is a randomized [quantum] public coin protocol for a function $f$ with communication $c$, error $\epsilon$, privacy loss $\delta$ against all distributions $\mu$ on inputs.

- For every distribution $\mu$ on inputs there is a deterministic [quantum] protocol for $f$ with communication $c$, error $\epsilon$, and privacy loss $\delta$ on that distribution.

Proof: The direction from randomized [quantum] to distributional deterministic [quantum] protocols follows by observing that a public coin protocol is really a probability distribution on deterministic [quantum] protocols, and for each distribution $\mu$ on the inputs the expected error (when picking a deterministic [quantum] protocol) is $\epsilon$, and likewise the expected privacy loss is $\delta$. Now due to the Markov inequality for each $\mu$ there must be one deterministic [quantum] protocol that has error at most $2 \epsilon$ and privacy loss at most $2 \delta$ simultaneously.

For the other direction assume the second statement holds, then combine error and privacy loss into one parameter by setting $\operatorname{para}(P, \mu)=$ $\operatorname{err}(P, \mu) \cdot \delta+\operatorname{loss}(P, \mu) \cdot \epsilon$, where $\operatorname{err}(P, \mu)$ denotes the error of a deterministic [quantum] protocol $P$ on $\mu$, and $\operatorname{loss}(P, \mu)$ the privacy loss. Note 
that we are guaranteed that for each $\mu$ there is a $P$ with $\operatorname{para}(P, \mu) \leq 2 \epsilon \delta$. Now the standard Yao principle gives us a single public coin randomized [quantum] protocol that has expected $\operatorname{para}(P, \mu) \leq 2 \epsilon \delta$ for all $\mu$. Such a protocol must have expected error at most $2 \epsilon$ and expected privacy loss at most $2 \delta$ for all $\mu$.

Our definition of communication complexity allows no public coins, however. If we are only interested in the privacy loss, one of the players may simply flip enough coins and communicate them to the other player, then they simulate the public coin protocol. This increases the communication, but none of the other parameters.

We need another result to get rid of the public coin at a lower cost in randomized protocols, if the leakage resp. privacy loss is very small. First consider the following lemma concerning leakage, proved completely analogous to the results in 28.

Lemma 8 Let $f:\{0,1\}^{n} \times\{0,1\}^{n} \rightarrow \mathbb{N}$ be computable by a randomized [quantum] protocol with error $\epsilon$, that uses public classical randomness and $c$ bits of communication and leaks $\delta$.

Then for all $\gamma>0$ there is a randomized [quantum] protocol for $f$ with error $(1+\gamma) \epsilon$, leakage $(1+\gamma) \delta$, and communication $O(c+\log n+\log (1 / \delta)+$ $\log (1 / \gamma)+\log (1 / \epsilon))$ that uses no public coin.

If leakage is small a bound on privacy loss is implicit.

Lemma 9 Let $f:\{0,1\}^{n} \times\{0,1\}^{n} \rightarrow \mathbb{N}$ be computable by a randomized [quantum] protocol (with error but using no public randomness) that has leakage $\delta \leq 1 /$ e.

Then the same protocol has privacy loss at most $n \cdot \delta-\delta \log \delta$.

Proof: First consider the case of classical protocols. Let $\mu$ be any distribution on the inputs. The distribution on the values of Bob's and Al's private storage $A, B$, when the inputs $X, Y$ are drawn according to $\mu$ is denoted $\rho_{A B X Y}$ (at some point in the protocol). If inputs are fixed to $x, y$ the resulting (normalized) distribution is denoted $\rho_{A B}^{x y}$. The leakage requirement states that for $x, y, y^{\prime}$ with $f(x, y)=f\left(x, y^{\prime}\right)$ we have $\left\|\rho_{A}^{x y}-\rho_{A}^{x y^{\prime}}\right\|_{1} \leq \delta$. For $x, y$ with $f(x, y)=z$ let

$$
\rho_{A}^{x}(z)=\sum_{y^{\prime}: f\left(x, y^{\prime}\right)=z} \frac{\mu\left(x, y^{\prime}\right)}{\sum_{a: f(x, a)=z} \mu(x, a)} \rho_{A}^{x y^{\prime}} .
$$

Then due to convexity for all $x, y$

$$
\left\|\rho_{A}^{x y}-\rho_{A}^{x}(f(x, y))\right\|_{1} \leq \delta .
$$


The continuity of entropy (fact 3) and taking the expectation over $x, y$ acording to $\mu$ then gives us

$$
E_{x, y}\left[H\left(\rho_{A}^{x}(f(x, y))\right)-H\left(\rho_{A}^{x y}\right)\right] \leq n \delta-\delta \log \delta .
$$

The left hand side equals

$$
H(A \mid X, f(X, Y))-H(A \mid X, Y, f(X, Y))=I(A: Y \mid X, f(X, Y)) .
$$

The leakage to Bob is analyzed in the same way. The quantum case is completely analogous.

Now we can say something about privacy loss and private coins.

Lemma 10 Let $f:\{0,1\}^{n} \times\{0,1\}^{n} \rightarrow \mathcal{Z}$ be computable by a randomized [quantum] protocol with error $\epsilon$ (using a public classical coin) and $c$ bits of communication, that has privacy loss $\delta \geq 1 / 2^{2 n}$.

Then there is a randomized [quantum] protocol for $f$ with error $2 \epsilon$, privacy loss $O(n \cdot \sqrt{\delta})$, and communication $O(c+\log n+\log (1 / \delta)+\log (1 / \epsilon))$ that uses no public coin.

Proof: Consider the case of classical protocols. Given the protocol, for, say, player $\mathrm{Al}$, all distributions and all rounds we have $I(A$ : $Y \mid X, f(X, Y)) \leq \delta$. Denote the distribution of the values of Al's register $\rho_{A}^{x y}$ for some inputs $x y$, let $\rho_{A}^{x}(z)$ denote the distribution in which $x$ and $z=f(x, y)$ are fixed, but $y$ is random. Then

$$
\begin{aligned}
& E_{z} E_{x, y: f(x, y)=z}\left\|\rho_{A}^{x y}-\rho_{A}^{x}(z)\right\|_{1} \\
= & E_{z} E_{x \mid z} E_{y \mid z x}\left\|\rho_{A}^{x y}-\rho_{A}^{x}(z)\right\|_{1} \\
\stackrel{*}{\leq} & E_{z, x} \sqrt{2 \ln (2) I(A: Y \mid X=x, f(X, Y)=z)} \\
\leq & \sqrt{2 \ln (2) E_{z, x} I(A: Y \mid X=x, f(X, Y)=z)} \text { with Jensen's inequality } \\
\leq & \sqrt{2 \ln (2) \delta}
\end{aligned}
$$

with $\left(^{*}\right)$ due to fact [5] where for a fixed $z, x$ the $y$ are coded (on the induced distribution) as $\rho_{A}^{x y}$ and the average code is $\rho_{A}^{x}(z)$. Hence

$$
E_{x, y, y^{\prime}: f(x, y)=f\left(x, y^{\prime}\right)} \mid\left\|\rho_{A}^{x y}-\rho_{A}^{x y^{\prime}}\right\|_{1} \leq 2 \sqrt{2 \ln (2) \delta} .
$$

Since this holds for all distributions, the same holds for all $x, y, y^{\prime}$ with $f(x, y)=f\left(x, y^{\prime}\right)$, thus the protocol leaks at most $2 \sqrt{2 \ln (2) \cdot \delta}$.

Invoking lemma 8 with $\gamma=1$ we get a protocol with the desired communication complexity and error, and leakage $O(\sqrt{\delta})$ using no public coin. Then an application of the previous lemma completes the proof for leakage at most $1 /$ e. For larger leakage the lemma is trivial.

The quantum case is completely analogous. 
Finally, the following lemma states that independent repetitions of a randomized protocol allow to decrease the error probability with a reasonable increase in privacy loss.

Lemma 11 Let $P$ be any randomized protocol for a function $f$ with error $1 / 3$, privacy loss $l$, and communication $c$.

Then there is a randomized protocol for $f$ with error $1 / 2^{k}$, privacy loss $O(k \cdot l)$, and communication $O(k \cdot c)$.

Proof: Repeat the protocol $t=O(k)$ times independently. By standard considerations taking the majority output yields the desired error bound and increases the communication as desired. We now show that the privacy loss is also as stated.

To see this consider the global state $\rho_{A B X Y}$, where Al's storage $A$ consists of $A_{1}, \ldots, A_{t}$ for the $t$ repetitions. W.l.o.g. $A_{i}$ contains a message history of the $i$ th repetition of the protocol. Note that $I\left(A_{i}: A_{j} \mid X=x, Y=y\right)=0$ for all $i \neq j$ and all $x, y$, if Al plays honest, since he is forced to send messages as in a completely new run of the protocol for all histories of the first $i$ repetitions, i.e., using fresh randomness. Then

$$
\begin{aligned}
& I\left(A_{1}, \ldots, A_{t}: Y \mid X=x, f(X, Y)=z\right) \\
= & H\left(A_{1}, \ldots, A_{t} \mid X=x, f(X, Y)=z\right) \\
- & H\left(A_{1}, \ldots, A_{t} \mid Y, X=x, f(X, Y)=z\right) \\
= & H\left(A_{1}, \ldots, A_{t} \mid X=x, f(X, Y)=z\right) \\
- & E_{y} H\left(A_{1}, \ldots, A_{t} \mid Y=y, X=x\right)
\end{aligned}
$$

for all $x, z$, with the expectation over $y \in Y$ under the distribution conditioned on $f(x, y)=z$. And due to the subadditivity of entropy this is at most

$$
\sum_{i} H\left(A_{i} \mid X=x, f(X, Y)=z\right)-E_{y} H\left(A_{1}, \ldots, A_{t} \mid Y=y, X=x\right) .
$$

The latter term equals

$$
E_{y} \sum_{i} H\left(A_{i} \mid Y=y, X=x\right),
$$

since for a fixed input $x, y$ the random variables $A_{i}$ are independent. So we get

$$
\begin{aligned}
& \sum_{i} H\left(A_{i} \mid X=x, f(X, Y)=z\right) \\
- & \sum_{i} E_{y} H\left(A_{i} \mid Y=y, X=x\right) \\
= & \sum_{i} I\left(A_{i}: Y \mid X=x, f(X, Y)=z\right) .
\end{aligned}
$$


Hence, with $I\left(A_{i}: Y \mid X, f(X, Y)\right)=E_{x, z} I\left(A_{i}: Y \mid X=x, f(X, Y)=z\right)$ :

$$
\begin{aligned}
& I\left(A_{1}, \ldots, A_{t}: Y \mid X, f(X, Y)\right) \\
\leq & \sum_{i} I\left(A_{i}: Y \mid X, f(X, Y)\right) \\
= & t \cdot I\left(A_{1}: Y \mid X, f(X, Y)\right) .
\end{aligned}
$$

\section{An exponential decrease in privacy loss}

In this section we give an example of a function that can be computed with an exponentially smaller privacy loss in the quantum case than in the classical case. This function is the disjointness problem, and the quantum protocol we consider is the protocol due to Buhrman, Cleve, and Wigderson given in [9]. In fact we describe a general way to protect a certain type of protocols against large privacy loss.

We now roughly sketch how the protocol works, and then how to make it secure. The protocol is based on a general simulation of black-box algorithms given in 9]. A black-box algorithm for a function $g$ is turned into a communication protocol for a function $g(x \wedge y)$ for the bitwise defined operation $\wedge$. The black-box algorithm for OR is the famous search algorithm by Grover [19] or rather its variant in [8]. The important feature of the protocol for us is that the players send a set of $\log n+O(1)$ qubits back and forth and apart from that no further qubits or classical storage depending on the inputs are used. Also the protocol runs in $O(\log n)$ stages, each concluded by a measurement. If this measurement yields an index $i$ with $x_{i}=y_{i}=1$, then the protocol stops (and rejects), else it continues. The qubits contain a superposition over indices $i$ from 1 to $n$ plus the values of $x_{i}$ and $x_{i} \wedge y_{i}$. So an honest player that does not attempt to get more information learns $O(\log n)$ times the measurement result for $O(\log n)$ qubits and thus an information of at most $O\left(\log ^{2} n\right)$.

The main tool to show that the privacy loss is small against players trying to get more information is the following generalization of the famous no-cloning theorem 31. While the no-cloning theorem says that we cannot make a perfect copy of an unknown quantum state (which would enable us to find out some information about the state without changing the original by measuring the copy), this lemma says that no transformation leaving two nonorthogonal originals both unchanged gives us any information about those states.

Lemma 12 Let $\left|\phi_{1}\right\rangle$ and $\left|\phi_{2}\right\rangle$ be two states that are nonorthogonal. Assume a unitary map $U$ sends $\left|\phi_{1}\right\rangle \otimes|0\rangle$ to $\left|\phi_{1}\right\rangle \otimes|a\rangle$ and $\left|\phi_{2}\right\rangle \otimes|0\rangle$ to $\left|\phi_{2}\right\rangle \otimes|b\rangle$. Then $|a\rangle=|b\rangle$.

Proof: The following simple proof has been proposed by Harumichi Nishimura [personal communication]. 
Note that the inner product of $\left|\phi_{1}\right\rangle$ and $\left|\phi_{2}\right\rangle$ is unchanged when we append some empty qubits, and when we apply the same unitary operation to the states. Hence

$$
\begin{aligned}
\left\langle\phi_{2} \mid \phi_{1}\right\rangle & =\left\langle\phi_{2}\left|\left\langle 0\left|U^{\dagger} U\right| \phi_{1}\right\rangle\right| 0\right\rangle \\
& =\left\langle\phi_{2}\left|\left\langle b \mid \phi_{1}\right\rangle\right| a\right\rangle=\left\langle\phi_{2} \mid \phi_{1}\right\rangle \cdot\langle b \mid a\rangle .
\end{aligned}
$$

Because $\left|\phi_{1}\right\rangle$ and $\left|\phi_{2}\right\rangle$ are nonorthogonal their inner product is nonzero and hence we have $\langle b \mid a\rangle=1$. Therefore, $|a\rangle=|b\rangle$.

Now assume a protocol sends $k$ qubits (in a pure state) back and forth without using any private storage whose state depends on the input and without measuring (this is what happens in the protocol for $D I S J_{n}$ during all $O(\log n)$ stages). If we manage to change the messages in a way so that for no inputs $x, x^{\prime}, y$ the message sent in round $t$ for input $x, y$ is orthogonal to the message for input $x^{\prime}, y$, then there is no transformation for Bob that leaves the message unchanged, yet extracts some information. In other words, honest players are forced to follow the protocol without getting further information. The only information is revealed at the end of a stage, when one player is left with the qubits from the last message, resp. at the time when one player decides to quit the protocol. Thus at most "size of the communication channel" (i.e., $k$ ) information is revealed.

We now describe how to make the messages nonorthogonal.

Lemma 13 For all $\epsilon>0$ and for any finite set of $l$-dimensional unit vectors $\left\{v_{i}\right\}$ there is a set of $l+1$ dimensional unit vectors $\left\{v_{i}^{\prime}\right\}$ such that $\left\|\tilde{v}_{i}-v_{i}^{\prime}\right\|<$ $\epsilon$, and $v_{i}^{\prime} \perp v_{j}^{\prime}$ for no $i, j$, where $\tilde{v}_{i}$ denotes $v_{i}$ with an appended 0 in dimension $l+1$.

Proof: For all vectors $v_{i}$ the vector $\tilde{v}_{i}$ is $l+1$ dimensional and contains the value 0 in the $l+1$ st dimension and the same values as $v_{i}$ in the other dimensions. Then change $\tilde{v}_{i}(l+1)$ to $\delta$, and scale all other values by $\sqrt{1-\delta^{2}}$ to obtain $v_{i}^{\prime}$. The resulting vectors have norm $\left\langle v_{i}^{\prime} \mid v_{i}^{\prime}\right\rangle=\delta^{2}+\left(1-\delta^{2}\right) \cdot\left\langle v_{i} \mid v_{i}\right\rangle=$ 1. The inner product of two vectors is $\left\langle v_{i}^{\prime} \mid v_{j}^{\prime}\right\rangle=\delta^{2}+\left(1-\delta^{2}\right)\left\langle v_{i} \mid v_{j}\right\rangle$. For a finite set of, say $k$, vectors there are $k^{2}$ different values of inner products $\left\langle v_{i} \mid v_{j}\right\rangle$. Using $\delta$ with $-\delta^{2} /\left(1-\delta^{2}\right)$ different from all these values and small enough that $\left\|v_{i}-v_{i}^{\prime}\right\| \leq \epsilon$ leads to a set of vectors with the desired properties.

We can state the upper bound for disjointness.

Theorem 1 DIS $J_{n}$ can be computed by a quantum protocol with error $1 / 3$, communication $O(\sqrt{n} \log n)$, and privacy loss $O\left(\log ^{2} n\right)$.

Proof: In [9] a quantum protocol with error $1 / 4$ and communication $O(\sqrt{n} \log n)$ is described, in which $\mathrm{Al}$ and Bob exchange pure state messages of length $\log n+O(1)$, but use no further storage depending on the 
input. The protocol consists of $O(\log n)$ stages each of which ends with a measurement of the qubits in the standard basis. No further measurements are used.

We modify the protocol. We add one more qubit to the messages. Then we change the first message to be sent (prepared by $\mathrm{Al}$ ) as described in lemma 13. The error introduced by this change is arbitrarily small. Then the protocol is used as before, ignoring the new qubit in all transformations, but always sending that qubit with the other qubits back and forth between the players. This can be done in a way ensuring that no message sent for any pair of inputs $x, y$ in any round will be orthogonal to another such message.

Assume Bob wants to get more information than he can get from the $O(\log n)$ classical strings of length $\log n+O(1)$ obtained from the measurements. In some round he will first start an attack on the message. He has to map the message received to another message he must send back. The second message is the result of a fixed unitary transformation (depending on $y$ ) on the first. He has to combine the attack with that unitary transformation. So we may assume that he first attacks the message and then applies the transformation to get the next message. The attack transformation maps the message and some empty qubits to the tensor product of the same message and another state, that depends on the other player's input. Lemma12 ensures that this is impossible. So Bob has to stick to the protocol without getting more information than allowed.

Now we turn to the lower bound. Every classical deterministic protocol partitions the communication matrix into rectangles labelled with the output of the protocol. Let $\mu$ be a distribution on the inputs. A labelled rectangle is $(1-\epsilon)$-correct, if according to $\mu$ at least $1-\epsilon$ of the weight of the rectangle is on correctly labelled inputs. Due to Yao's lemma a randomized protocol with error $\epsilon$ and communication $c$ yields for every distribution $\mu$ a deterministic protocol that has error $\epsilon$ and the same communication. Such a protocol induces a partition of the communication matrix into $2^{c}$ rectangles with overall error $\epsilon$.

The width of a rectangle $A \times B$ is $\min \{|A|,|B|\}$. Let $r(f)$ denote the largest width of any completely correct rectangle. 4] proves:

Fact $14 C L_{0}(f) \geq(n-\log r(f)) / 2-1$ for all $f:\{0,1\}^{n} \times\{0,1\}^{n} \rightarrow\{0,1\}$.

We now describe a new bound. An $a$-rectangle is a rectangle that contains predominantly the function value $a$. The maximum size of a $(1-\epsilon)-$ correct $a$-rectangle according to $\mu$ is called $s_{\epsilon}^{a}(f, \mu)$. Let uni denote the uniform distribution.

Lemma 15 Choose $a \in \mathcal{Z}$. All randomized protocols with error $1 / 3$ computing $f:\{0,1\}^{n} \times\{0,1\}^{n} \rightarrow \mathcal{Z}$ have privacy loss

$$
\Omega\left(\frac{u n i\left(f^{-1}(a)\right) \cdot \log \left(1 / s_{1 / n^{2}}^{a}(f, \text { uni })\right)}{\log n}-O(1)\right) .
$$


Proof: Given a randomized protocol with error $1 / 3$ and privacy loss $c$ we can find a protocol with privacy loss $c \cdot k$ and error $1 / 2^{\Omega(k)}$ by repeating the protocol with independent coin flips $k$ times and taking the majority output due to lemma 11 .

For $k=O(\log n)$ we get a randomized protocol with privacy loss $l / 2=$ $O(c \log n)$, and error $1 /\left(2 n^{4}\right)$. This privacy loss is guaranteed against all distributions, and by one side of lemma 7 for the uniform distribution $u n i$ there is a deterministic protocol that has the privacy loss $l$, and error $1 / n^{4}$ on uni. The deterministic protocol corresponds to a partition of the communication matrix into rectangles with global error $1 / n^{4}$, so $1-1 / n^{2}$ of all inputs are in rectangles that are $\left(1-1 / n^{2}\right)$-correct. Each $\left(1-1 / n^{2}\right)$-correct $a$-rectangle has weight at most $s=s_{1 / n^{2}}^{a}(f, u n i)$. Furthermore the total weight of inputs with function value $a$ in other rectangles is at most $\alpha=1 / n^{4}$.

After running the protocol we have a distribution on the values of Al's storage $A$ and Bob's storage $B$. W.l.o.g. both players have stored the complete message history as a string $m$. Such a string is a label to a rectangle in the communication matrix. Call such a rectangle $U_{m} \times V_{m}$ and let $h(m)$ denote the height $\left|U_{m}\right| / 2^{n}$, let $b(m)$ denote the base $\left|V_{m}\right| / 2^{n}$. $1-1 / n^{2}$ of all inputs are in $\left(1-1 / n^{2}\right)$-correct rectangles. Let $M_{a}$ denote the set of $\left(1-1 / n^{2}\right)$-correct $a$-rectangles/message sequences in which the protocol outputs $a . \operatorname{Pr}(m)$ denotes the probability of rectangle $m$, i.e., its size under the uniform distribution.

The inputs that are not in $\left(1-1 / n^{2}\right)$-correct $a$-rectangles but have function value $a$ have weight at most $\beta=1 / n^{4}+1 / n^{2}<2 / n^{2}$. They can contribute at most $\gamma=\beta n-\beta \log \beta=o(1)$ to an entropy due to fact 4 . Then

$$
\begin{aligned}
& H(Y \mid A, X, f(X, Y)=a)+H(X \mid B, Y, f(X, Y)=a) \\
= & \sum_{m} \operatorname{Pr}(m)[H(Y \mid m, X, f(X, Y)=a)+H(X \mid m, Y, f(X, Y)=a)] \\
\leq & \gamma+\sum_{m \in M_{a}} \operatorname{Pr}(m)\left[\log \left(2^{n} \cdot b(m)\right)+\log \left(2^{n} \cdot h(m)\right)\right] \\
\leq & o(1)+\sum_{m \in M_{a}} \operatorname{Pr}(m) \log \left(\left|V_{m}\right| \cdot\left|U_{m}\right|\right) \\
\leq & o(1)+\sum_{m \in M_{a}} \operatorname{Pr}(m) \log \left(2^{2 n} s\right) \\
\leq & 2 n+\log s+o(1) .
\end{aligned}
$$

Also, assume that $u n i^{-1}(a) \geq 1 / n^{2}$, then

$$
H(Y \mid X, f(X, Y)=a) \geq n-4 \log n-o(1),
$$

since in this case only $1 / n^{2}$ of the weight of the distribution that is uniform on inputs with $f(x, y)=a$ can lie on rows $x$ having less than $2^{n} / n^{4}$ 
columns $y$ with $f(x, y)=a$. So if we pretend that all $x$ have at least $2^{n} / n^{4}$ corresponding $y$ with $f(x, y)=a$, we increase the actual entropy by at most $1 / n^{2} \cdot n=o(1)$. But this would lead to $H(Y \mid X, f(X, Y)=a) \geq \log \left(2^{n} / n^{4}\right)$.

Consequently

$\operatorname{uni}\left(f^{-1}(a)\right) \cdot H(Y \mid X, f(X, Y)=a) \geq \operatorname{uni}\left(f^{-1}(a)\right) \cdot(n-4 \log n)-o(1)$.

This gives us

$$
\begin{aligned}
& O(c \log n) \\
\geq & I(Y: A \mid X, f(X, Y))+I(X: B \mid Y, f(X, Y)) \\
\geq & \operatorname{uni}\left(f^{-1}(a)\right) \cdot[I(Y: A \mid X, f(X, Y)=a)+I(X: B \mid Y, f(X, Y)=a)] \\
= & \operatorname{uni}\left(f^{-1}(a)\right) \cdot[H(Y \mid X, f(X, Y)=a)+H(X \mid Y, f(X, Y)=a) \\
& -H(Y \mid A, X, f(X, Y)=a)-H(X \mid B, Y, f(X, Y)=a)] \\
\geq & \operatorname{uni}\left(f^{-1}(a)\right) \cdot[2 n-8 \log n-2 n-\log s]-o(1) \\
\geq & \operatorname{uni}\left(f^{-1}(a)\right) \cdot(-\log (s))-O(\log n) .
\end{aligned}
$$

The following is proved in [2]

Fact 16 Let $\mu$ be the uniform distribution on pairs of sets of size $\sqrt{n}$ from a size $n$ universe. Then the largest $(1-\epsilon)$-correct 1-rectangle for disjointness (i.e., one that contains mostly disjoint pairs of sets) has size $1 / 2^{\Omega(\sqrt{n})}$ for some constant $\epsilon$.

Corollary $1 C L_{1 / 3}\left(D I S J_{n}\right)=\Omega(\sqrt{n} / \log n)$.

\section{The class of private functions}

We have seen in the previous section that certain functions can be quantum computed with less privacy loss against honest players than possible in the classical case. In this section we show that, however, the class of functions which can be computed privately (i.e., with privacy loss 0 ) is unchanged by allowing quantum communication, if we consider honest players (i.e., those who are not trusted to continue with the protocol until the end).

\subsection{Players that do not preempt}

But first let us take a look at the model of honest players, in which only the information retrievable at the end of the protocol is counted.

Theorem 2 For every function $f$ with deterministic communication complexity $c$ there is a quantum protocol with communication $O\left(c^{2}\right)$, where the 
final state obtainable by every honest player has an arbitrarily small distance to the final state of a player that knows only his input and the function value at the end.

Thus we get an arbitrarily close approximation of privacy against honest players if we consider only the information available at the end of the protocol. In other words if we trust the other player not only to play honest, but also to not quit before the end of the protocol, every function can be computed in a secure way.

Proof: Suppose there is a deterministic protocol for $f$ with complexity c. First we turn this into a protocol, in which the players do not need to store anything besides the current message, i.e., they compute the new message from the message they received, send the message, and remember nothing else. For this the players simply exchange a complete message history in all the rounds, increasing the complexity to $c^{2}$ at most. Now following lemma 13 we can turn this into a quantum protocol with arbitrary small error $\epsilon$ and communication $c^{2}+c$, in which only pure state messages are exchanged, so that for no inputs $x, y, y^{\prime}$ the messages on $x, y$ and on $x, y^{\prime}$ sent to $\mathrm{Al}$ (or $\mathrm{Bob}$ ) in some round $t$ are orthogonal. With lemma 12 then an honest $\mathrm{Al}$ cannot obtain information from the message he holds without changing the message similarly to the proof of theorem 1 thus Al has to send the message and is left with no information in all rounds after the message is sent.

At the end, however a complete message history is available to one player, making the protocol highly nonprivate. To remove this problem consider the following. A clean protocol [15] is a protocol, in which the final state is

$$
|0\rangle|x\rangle|f(x, y\rangle| y\rangle|0\rangle \text {. }
$$

[15] shows how to transform any quantum protocol with error $\epsilon$ into a protocol, whose final state has distance $O(\sqrt{\epsilon})$ from the final state a clean protocol would have. We use this transformation, which also increases the communication complexity by a factor of 2 only and does not change the error (the idea is that the "garbage" produced by the computation is removed by "reversing" the protocol).

Thus we get a protocol with error $O(\sqrt{\epsilon})$, communication $O\left(c^{2}\right)$, in which in all rounds the players exchange a certain set of qubits, about which both players cannot obtain additional information, since these messages are pairwise nonorthogonal. In the end the state has arbitrarily small nonzero distance to a state revealing no additional information.

Due to the continuity of entropy described by fact 3 both the (informationtheoretically measured) privacy loss and the (distance measured) leakage can be made arbitrarily small at the end of a protocol. 


\subsection{The characterization of quantum privacy}

Now we return to our regular definition of privacy and show that here quantum communication does not enlarge the set of private functions. The set of classically private functions has been characterized in [23] and [5]. We extend this characterization to the quantum case.

Definition 1 Let $M=C \times D$ be a matrix. A relation $\equiv$ is defined as follows: rows $x$ and $x^{\prime}$ satisfy $x \equiv^{\prime} x^{\prime}$, if there is a column $y$ with $M_{x, y}=$ $M_{x^{\prime}, y}$. Then $\equiv$ is the transitive closure of $\equiv^{\prime}$. Similar relations are defined for columns.

A matrix is called forbidden, if all its rows are equivalent, all its columns are equivalent, and the matrix is not monochromatic.

Theorem 3 If the communication matrix of $f$ contains a forbidden submatrix then $f$ cannot be computed by a quantum protocol with error smaller than 1/2 and no privacy loss.

Proof: A quantum protocol with error smaller than $1 / 2$ for $f$ must also solve the problem $g$ corresponding to the forbidden submatrix. If $A$ contains the row-labels and $B$ the column labels of the forbidden submatrix then $g$ is defined on $A \times B$ and $g(x, y)=f(x, y)$. This problem $g$ is nontrivial, since the submatrix is not monochromatic. Suppose a given protocol computes $g$ with error smaller than $1 / 2$ and privately. We will show that one round after the other can be shaved off the protocol, eventually yielding a protocol for $g$ with one round. Such a protocol cannot compute $g$ with error smaller than $1 / 2$, thus we reach a contradiction.

We show that the first message (w.l.o.g. sent by $\mathrm{Al}$ ) does not depend on the input, and can thus be computed by Bob, whereupon the first round of communication can be skipped. Let $x_{1}, \ldots, x_{l}$ denote the rows of the forbidden submatrix, enumerated in such a way that $x_{i} \equiv^{\prime} x_{j}$ for some $j<i$ for all $i>1$. If $x_{i} \equiv^{\prime} x_{j}$ then there is a $y$, so that $g\left(x_{i}, y\right)=g\left(x_{j}, y\right)$. Since it is possible that Bob holds $y, \mathrm{Al}$ is not allowed to send different messages on $x_{i}$ and $x_{j}$, since otherwise Bob may obtain information about the identity of Al's inputs $x_{i}$ and $x_{j}$ not deducible from the function value alone. So for all $x_{i}$ the same message is sent.

Let $\rho_{A M}^{x y}$ denote the state of Al's qubits right before the first message is sent (on inputs $x, y$ ), with $M$ containing the message. $\rho_{M}^{x y}$ is the same for all $x, y$. Also $\rho_{A M}^{x y}$ purifies such a state. Due to the local transition theorem (fact 6) $\mathrm{Al}$ has unitary operations acting on register $A$ that switch between those states (for different $x$ ) without introducing any error. Thus Bob may

prepare $\rho_{A M}^{x, y}$ for some fixed $x$, send the part of the state in $A$ to $\mathrm{Al}$ and keep the $M$ part. Al can then change the received state to the one for the correct $x$. Furthermore Bob can send the message for round 2 together with the first message, thus we get a protocol with one round less. 
Repeating this we eventually arrive at a protocol with one round only, in which, say, a message is sent from $\mathrm{Al}$ to Bob. Thus if the error is smaller than $1 / 2$ the output does not depend on $y$. Consequently the communication matrix of $g$ consists of monochromatic rows only, and there are at least two different such rows, since the matrix is not monochromatic. Such a matrix is clearly not a forbidden submatrix, since two different monochromatic rows are not equivalent. Thus we arrive at a contradiction to our assumptions on $f$ or on the protocol.

Now that we know a forbidden submatrix excludes a private quantum protocol, the other piece for a characterization is as follows, see 23 .

Fact 17 If the communication matrix of $f$ contains no forbidden submatrix, then $f$ can be computed by a deterministic private protocol.

Thus the class of privately computable functions is invariant under the choice of quantum or classical communication.

A function $f$ can be computed with privacy loss $k$ in the hint sense, if there is a privately computable function $h$, such that $f(x, y)$ can be computed from $h(x, y)$, and $k=\log (\operatorname{range}(h))-\log (\operatorname{range}(f))$. Since a function $h$ can be computed privately deterministically, iff $h$ can be computed privately by a quantum protocol, we get the following.

Corollary 2 The privacy loss of a function $f$ in the hint sense is unchanged if we allow quantum protocols.

The structure imposed on protocols by the privacy constraint is actually strong enough to deduce a lower bound on the number of rounds needed to compute a function.

Theorem 4 Any function $f$ computable by a private quantum protocol with error smaller than 1/2 and $r$ rounds of communication can also be computed by a private deterministic protocol with no error using at most $r$ rounds.

Proof: We construct a protocol tree from the quantum protocol. This is a layered directed tree whose vertices are indexed with rectangles in the communication matrix. Rectangles $A \times B$ in depth $d$ have children $A_{i} \times B$ with disjoint $A_{i}$ covering $A$, or children $A \times B_{i}$ with disjoint $B_{i}$ covering $B$. In depth $d$ either all edges lead to vertices that decompose the set of rows or all edges lead to vertices that decompose the set of columns.

The root is indexed by the communication matrix $M=A \times B$ of $f$. W.l.o.g. assume $\mathrm{Al}$ sends a message in the first round. Then the set of messages used by $\mathrm{Al}$ decomposes the set of rows into disjoint subsets. Note that if $x \equiv x^{\prime}$ for two inputs $x, x^{\prime}$ to Al then these inputs share the same message in the first round. Recall that a message is in general a mixed quantum state. If Al's messages induce subsets $A_{1}, \ldots, A_{t}$ of the rows, and 
the equivalence relation $\equiv$ on rows (relative to $M$ ) has equivalence classes $C_{1}, \ldots, C_{l}$ then each $C_{i} \subseteq A_{j}$ for exactly one $j$.

Now from the point of view of Bob all rows in some set $A_{i}$ are equivalent when he sends his message in round 2 . Hence we may as before decompose the columns of each rectangle $A_{i} \times B$ according to the messages used by Bob. Again any equivalence class $C_{i}$ for columns (where the equivalence relation $\equiv$ is chosen relative to $A_{i} \times B$ ) lies in exactly one subset $B_{j}$ of the row decomposition induced by Bob's messages.

In this manner we can inductively follow the protocol round per round to find a protocol tree. Note that all inputs in the rectangles attached to the leaves of the tree have the same acceptance probabilities, which are either all smaller than $1 / 2$, or all larger than $1 / 2$. Hence it is true that the rectangles attached to leaves are monochromatic.

A protocol tree trivially induces a deterministic protocol for $f$ using as many rounds as the tree is deep, while the depth of the constructed tree is the number of rounds of the quantum protocol.

Since the deterministic protocol constructed for the previous theorem trivially doesn't have to communicate more than $n$ bits in one round, its communication cost is at most $n$ times higher than the number of rounds of the quantum protocol, which is a lower bound on the quantum communication cost.

Corollary 3 The communication cost of an optimal private quantum protocol for a function $f:\{0,1\}^{n} \times\{0,1\}^{n} \rightarrow \mathbb{N}$ is at most a factor $n$ smaller than the communication cost of an optimal private deterministic protocol for $f$.

\subsection{Boolean functions and leakage}

Next we consider the case of Boolean functions. It is known 14 that the class of private Boolean functions is the class of functions $f_{A}(x) \oplus f_{B}(y)$, even if one considers protocols that leak $\delta$ (recall that this refers to the distance sense of leaking) and have error $\epsilon$ with $\epsilon+\delta<1 / 2$. These functions are combinatorially characterized by the so-called "corners lemma" [14 saying that there is no $2 \times 2$ rectangle in the communication matrix containing 3 ones and 1 zero or vice versa. As a corollary of theorem 3 we get a result for the quantum case with no leakage.

Corollary 4 If the communication matrix of a function $f$ contains a $2 \times$ 2 rectangle with exactly 3 times the same entry, then no private quantum protocol with error smaller than $1 / 2$ can compute $f$.

Corollary 5 The class of Boolean functions computable by private quantum protocols is the class of functions $f_{A}(x) \oplus f_{B}(y)$. 
Is corollary 4 also valid in the quantum case with small leakage? The answer is no. There are function satisfying the assumptions of the corners lemma which can be computed with small leakage by a quantum protocol.

Theorem 5 There is a quantum protocol computing the AND function on two bits with error $1 / 3$ that has leakage $\delta$ and uses $O\left(1 / \delta^{2}\right)$ communication.

Proof: We describe a protocol in which only $\delta$ is leaked to Bob, and nothing is leaked to Al. During $O\left(1 / \delta^{2}\right)$ rounds Al prepares a superposition $\delta / 2|00\rangle+\sqrt{1-\delta^{2} / 4}|11\rangle$ if $x=0$, and $\delta / 2|10\rangle+\sqrt{1-\delta^{2} / 4}|11\rangle$ if $x=1$. Note that the trace distance between the corresponding density matrices is $\delta$ due to equation (1). Thus if Bob receives such a message leakage to him is $\delta$. Bob then adds a blank qubit and if $y=1$ applies a unitary transformation that sends $|000\rangle$ to $|000\rangle,|100\rangle$ to $|101\rangle$, and $|110\rangle$ to $|110\rangle$. If $y=0$ he leaves the state unchanged. Then Bob sends the 3 qubits back to $\mathrm{Al}$. Al and Bob repeat this $O\left(1 / \delta^{2}\right)$ times with fresh qubits. In the end $\mathrm{Al}$ measures all triples in the standard basis. If he receives a $|000\rangle$ state he outputs 0 , if he gets a $|101\rangle$ state he outputs 1 (and sends the result to Bob). If he has no such results he gives up without answer.

Note that with probability $\delta^{2} / 4 \mathrm{Al}$ gets one of the desired results, thus $O\left(1 / \delta^{2}\right)$ experiments suffice to yield a protocol with constant error.

The leakage can be analyzed as follows. Suppose $x=y=1$. In this case there can be no leakage, since an input and the function value give away the other input.

Now suppose $x=1, y=0$. In this case there can be no leakage to Al. The leakage to Bob is $\delta$ given the message of a round. Due to lemma 12 Bob cannot get any information out of a message without becoming dishonest, since no two messages are mutually orthogonal. Thus for all rounds the information leaked to Bob is $\delta$.

Suppose $x=0$. Al always simply gets his message back, no matter what Bob's input is, so there is no leakage to Al. If $y=1$ then there can be no leakage to Bob. Otherwise the leakage to Bob is $\delta$ as above.

The communication complexity in the above construction is within a polynomial of the optimum.

Theorem 6 If the communication matrix of a function $f$ contains a $2 \times 2$ rectangle with exactly 3 times the same entry, then no quantum protocol with error $1 / 3$, leakage $\delta$, and at most $1 /(12 \sqrt{\delta})$ rounds can compute $f$.

Proof: A protocol containing the described submatrix can easily be adapted to compute the Boolean AND function on input bits $x, y$ with the same parameters. We show that the above stated number of rounds is necessary.

Let $\rho_{A M}^{x}$ denote the state of the private qubits of $\mathrm{Al}$ in $A$ and of the first message sent to Bob in $M$ in round one when Al's input is $x$. Then 
the given leakage demands that $\left\|\rho_{M}^{1}-\rho_{M}^{0}\right\|_{\mathrm{t}} \leq \delta$, since it is possible that Bob's input is $y=0$. The states $\rho_{A M}^{1}$ and $\rho_{A M}^{0}$ are purifications of the two states on the message qubits.

Due to the local transition theorem (fact 6) there is a unitary transformation $U$ on Al's qubits alone that maps $\rho_{A M}^{0}$ to a state $\tilde{\rho}_{A M}^{0}$ with

$$
\left\|\tilde{\rho}_{A M}^{0}-\rho_{A M}^{1}\right\|_{\mathrm{t}} \leq 2 \sqrt{\delta}
$$

We modify the protocol by skipping the first round. Instead Bob creates a state $\rho_{A M}^{0}$ by himself and sends the $A$ part to $\mathrm{Al}$ together with the communication of round 2. If Al's input is 0 , the protocol can continue without problems. If Al's input is 1 , he applies the unitary transformation $U$, which leads to the state $\tilde{\rho}_{A M}^{0}$ with distance $2 \sqrt{\delta}$ from $\rho_{A M}^{1}$. So the error introduced is at most $2 \sqrt{\delta}$ and the protocol runs with one round less.

Repeating the above process for a $k$ round protocol leads eventually to a protocol in which, say, $\mathrm{Al}$ sends one message and Bob none, so the output does not depend on Bob's input anymore. This can only happen when the error is at least $1 / 2$, so $1 / 3+(k-1) \cdot 2 \sqrt{\delta} \geq 1 / 2$, hence $k>1 /(12 \sqrt{\delta})$.

\section{$5 \quad$ Trading privacy loss against complexity}

In this section we show that allowing a privacy loss of much less than one bit (instead of privacy loss 0) can reduce the communication complexity of a certain function, namely the identified minimum problem, from exponential to polynomial. Thus protocols obtaining a very close approximation of privacy can be much cheaper than truly private protocols.

Theorem 7 The function IdMin ${ }_{n}$ can be computed by a randomized protocol with privacy loss $\delta$, error $\delta$, and communication $O\left(n^{3} / \delta \cdot \log (1 / \delta)\right)$.

Every quantum or randomized protocol computing IdMin $n_{n}$ with error $\epsilon<1 / 2$ and with privacy loss 0 needs communication $\Omega\left(2^{n}\right)$.

Proof: It is shown in 23] that any private randomized or deterministic protocol for $\operatorname{IdMin}_{n}$ needs $2 \cdot\left(2^{n}-1\right)$ communication rounds. With theorem 4 this implies that also quantum protocols need that many rounds. The communication cost is always at least as large as the number of rounds.

For the upper bound we proceed as follows. We first show that the function can be computed efficiently with small leakage. Then we invoke lemma 9 to get the result for privacy loss. To construct a protocol with small leakage we describe for every probability distribution on the inputs a deterministic protocol that has small expected leakage (over the input distribution). Using Yao's lemma like in lemma[7 we get a single randomized protocol that has small expected leakage against all distributions, where the expectation is over the coins of the protocol. Such a protocol immediately 
has small leakage in the sense of our standard definition. To remove the necessity of allowing public coin randomness we use lemma 8 . Then we are ready to guarantee also small privacy loss using lemma 9 ,

The expected leakage to $\mathrm{Al}$ in a protocol for a distribution $\mu$ is

$$
E_{x, y, y^{\prime}: f(x, y)=f\left(x, y^{\prime}\right)} \mid\left\|\rho_{A}^{x y}-\rho_{A}^{x y^{\prime}}\right\|_{1},
$$

for the state $\rho_{A B}^{x y}$ of the storage of $\mathrm{Al}$ and Bob in some round $t$. The expected leakage of the protocol is the maximum over all rounds and over Al,Bob.

The corresponding Yao lemma is as follows, being proved completely analogous to lemma 7 . Note that if a randomized protocol has for all inputs an expected leakage of at most $\zeta$ (with the expectation over its coins), then it has leakage $\zeta$ in the ordinary definition.

Lemma 18 The following statements are equivalent in the sense that if one is true for some values $\epsilon, \zeta$, then the other is true with values $2 \epsilon, 2 \zeta$.

- There is a randomized public coin protocol for a function $f$ with communication $c$, error $\epsilon$, and leakage $\zeta$.

- For every distribution $\mu$ on inputs there is a deterministic protocol for $f$ with communication $c$, and error $\epsilon$ and expected leakage $\zeta$ on $\mu$.

We start by describing a protocol with small expected leakage for the uniform distribution and then show how to adapt this protocol to an arbitrary distribution.

The protocol is defined inductively. For $n=O(1)$ we use the simple protocol with leakage 0 , in which $\mathrm{Al}$ asks for $z=1, \ldots, x-1$, whether $z \geq y$. If so for one $z$, then $2 y$ is the result, otherwise $2 x+1$ is the result. The protocol needs communication $O(1)$.

For larger $n$ we do the following. Let $\gamma=\delta /(16 n)$. Al asks Bob for all $z_{i}=\left\lceil(1+\gamma)^{i}\right\rceil<\min \left\{x, 2^{n-1}\right\}$ (with $i \in \mathbb{N}$ ), whether $z_{i} \geq y$. If so for one $z_{i}$, then $2 y$ is the output (given by Bob). Else, when $x \leq 2^{n-1}$, then $2 x+1$ is given as output. If this is not the case, then both players know the minimum is larger than $2^{n-1}$, and the protocol can be invoked recursively for $n-1$.

First we compute the communication cost of the protocol. Obviously the communication before the recursion is in $\log _{1+\gamma} 2^{n}=O(n / \gamma)=O\left(n^{2} / \delta\right)$ rounds, each communicating at most $n$ bits. So the recursion for the overall communication is $C(n)=O\left(n^{3} / \delta\right)+C(n-1)$. After $\log (1 / \delta)$ recursions, however, the remaining pairs of inputs have weight at most $\delta^{2}$. So we may stop there, and hence the communication is at most $O\left(n^{3} / \delta \cdot \log (1 / \delta)\right)$.

Next we compute the leakage and error of the protocol. Let $\alpha=(1+$ $\gamma)^{i} \leq x \leq(1+\gamma)^{i+1}<2^{n-1}$. Also assume that we are in an iteration, where the remaining input length is $n$ (which is not necessarily the original input 
length). If the protocol stops early, the error is at most $\delta^{2}$, and the leakage is 0 . Otherwise the following holds.

If $y \geq x$, then the protocol will neither err nor leak information.

If $\alpha \leq y<x$, then the protocol will err and will leak, since Bob learns $x$. This happens with probability at most

$$
\left((1+\gamma)^{i+1}-(1+\gamma)^{i}\right) / 2^{n}=\gamma(1+\gamma)^{i} / 2^{n} \leq \gamma / 2,
$$

and the leakage contributed by this case is at most $2 \cdot \gamma / 2=\delta /(16 n)$, the error at most $\gamma / 2$.

If $y<\alpha$, then the protocol is correct. There is, however, some leakage. Bob learns that $x>(1+\gamma)^{i}$ for some $i$, instead of just learning that $x>y$. This corresponds to Bob knowing that $x$ is distributed uniformly over all values larger than $(1+\gamma)^{i}$ instead of Bob knowing that $x$ is distributed uniformly over all values larger than $y$. The first distribution $p$ is uniform on $2^{n}-(1+\gamma)^{i}=2^{n}-(1+\gamma)^{i-1}-\gamma(1+\gamma)^{i-1}$ values, the second distribution $q$ is uniform on $2^{n}-y$ values. The distance between $p$ and $q$ is at most the distance between $p$ and the distribution $q^{\prime}$ in which $x$ is uniform over all values larger than $(1+\gamma)^{i-1}$. Then $q^{\prime}$ is uniform on $2^{n}-(1+\gamma)^{i-1}$ points. Since $(1+\gamma)^{i-1} \leq 2^{n} / 2$, the distance is at most $2 \gamma$ and the probability of this event is at most $1 / 2$, so the leakage contributed by this case is at most $\gamma=\delta /(16 n)$.

Thus the overall leakage is smaller than $\delta /(8 n)$, and the error $\gamma / 2+\delta^{2}<$ $\delta / 4$.

After describing the protocol for the uniform distribution we now turn to protocols for arbitrary distributions $\mu$. Since Al plays the role of an interrogator in the above protocol, while Bob only answers, let $x$ be an arbitrary input for $\mathrm{Al}$ and $\mu_{x}$ the induced distribution on Bob's inputs. Note that this distribution is known to $\mathrm{Al}$.

Let $r_{l}$ be the least integer satisfying $\sum_{i=1}^{r_{l}} \mu_{x}(i) \geq l / 2^{n}$ for $l=1, \ldots, 2^{n}$. The protocol proceeds as in the protocol for the uniform distribution, but $\mathrm{Al}$ queries $r_{l}$ instead of $l$ all the time, i.e., for $l=(1+\gamma)^{i}<\min \left\{x, r_{2^{n-1}}\right\}$.

The communication complexity of the protocol is still $O\left(n^{3} / \delta \cdot \log (1 / \delta)\right)$.

Let $\alpha=r_{(1+\gamma)^{i}} \leq x \leq r_{(1+\gamma)^{i+1}}$.

If $y \geq x$, then the protocol will neither err nor leak information.

If $\alpha<y<x$, then the protocol will err and will leak information. The probability that this happens is at most $(1+\gamma)^{i+1} / 2^{n}-(1+\gamma)^{i} / 2^{n} \leq$ $\gamma \frac{(1+\gamma)^{i}}{2^{n}} \leq \gamma / 2$, and the leakage in this case is at most $\gamma$, the error at most $\gamma / 2$.

If $y<\alpha$, then the protocol is correct, but again there is leakage. Bob gets to know that $x \geq \alpha$ instead of just knowing $x>y$, i.e., the (normalized) distribution on values larger than $y$ against the (normalized) distribution on values larger $\alpha$. The distance between these two distributions is at most $2 \gamma$. Thus the contribution to the expected leakage is at most $\gamma$. 
The expected leakage of all cases together is at most $\delta /(8 n)$.

So we get for every distribution a deterministic protocol with error $\delta / 4$ and expected leakage $\delta /(8 n)$. Then lemma 18 gives us a single public coin randomized protocol with leakage $\delta /(4 n)$. The communication complexity is $O\left(n^{4} / \delta\right)$, the error is at most $\delta / 2$.

Applying lemma 8 gives us a protocol with leakage $\delta /(2 n)$, error $\delta$, communication $O\left(n^{4} / \delta\right)$ using no public coin. Then we can use lemma 9 to see that the protocol actually has privacy loss at most $\delta$.

\section{Conclusions and open problems}

In this paper we have discussed privacy with respect to honest players with a focus on the themes quantum communication and protocols with (small) privacy loss or leakage. We have given an example of a function that can be computed with exponentially smaller privacy loss using quantum communication than in the case of classical communication. The set of functions with privacy loss 0 is, however, not enlarged by quantum communication. For Boolean functions we were able to give a simple characterization of the quantum private functions as $f_{A}(x) \oplus f_{B}(y)$. It is known [14 that allowing small leakage (leakage $\delta$ and error $\epsilon$ with $\epsilon+\delta<1 / 2$ ) for classical communication does not allow to compute more functions. In the quantum case, however, leakage allows to compute the AND function (with a tradeoff between the number of rounds and the leakage).

The characterization for Boolean functions can be extended to the case of multiparty private computation. As in [14 it can be shown that only functions of the form $f_{1}\left(x_{1}\right) \oplus \cdots \oplus f_{k}\left(x_{k}\right)$ can be quantum computed in a way so that every set of $\lceil k / 2\rceil$ players learns nothing more about the other players' inputs than what is deducible from their inputs and the output alone. Since every function can be computed classically so that no coalition of less than $k / 2$ players learns more than allowed [7, 12], and the aforementioned functions are private against coalitions of even $k-1$ players, there are only 2 levels in this hierarchy of privacy for quantum computation, as in the classical case, see [14. Note that more such levels exist for non-Boolean functions [13].

We now give some open problems. A more realistic type of player is an adversary that has two objectives: with highest priority he wants the output to be correct with large probability. But then he also wants to learn as much as possible under this constraint. As an illustration of the power of this kind of player consider a technique from a proof for a lower bound on the quantum communication complexity of the inner product function in [15]. Given any clean (for simplicity assume errorless) protocol for the inner product function, one player may take a uniform superposition over all possible inputs instead of his real input and use that protocol. Applying 
a Hadamard transform to his (fake) input register after the protocol has stopped supplies the player with the other player's input. So he is able to compute the function value and learn maximal information at the same time given any clean protocol. Note that the player is not even approximately honest, though.

A restricted form of this hard to analyze type of player is an almost honest player that roughly follows the protocol, but only sends messages that are in distance $\epsilon$ from the "correct" messages. This allows in the quantum case e.g. to use approximate cloning as in [10, or generally the following type of attack: the player uses the protocol with some probability $\epsilon$ for a fake input. Then he learns some information he should not know with probability $\epsilon$. If we measure the divulged information in the information theoretic sense there are private functions, e.g. IdMin ${ }_{n}$, for which such a player can obtain $\epsilon n$ bits of information while being approximately honest.

A study of privacy with approximately honest players would be interesting. In particular, can the quantum protocol for disjointness be made secure against them? The set of private Boolean functions is robust against such players, since they are of the form $f_{A}(x) \oplus f_{B}(y)$. About non-Boolean functions no results seem to be known even in the classical case.

Another open problem is the following: can we extend the characterization of (non-Boolean) classically private functions for the case of small leakage, or does small leakage make some (non-Boolean) nonprivate functions computable in the classical case?

Finally, how can one prove lower bounds on the privacy loss of quantum protocols? Since the privacy loss is always smaller than the communication complexity, this is different from proving lower bounds for quantum communication complexity as e.g. in 21, 30. We have shown that the quantum privacy loss of the disjointness problem is only $O\left(\log ^{2} n\right)$, while the quantum communication complexity of disjointness is $\Omega(\sqrt{n})$ [30].

\section{Acknowledgements}

The author wishes to thank Harumichi Nishimura for useful comments improving the presentation of lemma 12, Hoi-Kwong Lo for interesting discussions, and the referees for useful comments.

\section{References}

[1] D. Aharonov, A. Kitaev, and N. Nisan. Quantum circuits with mixed states. 30th ACM Symposium on Theory of Computing, pp. 20-30, 1998. Also: quant-ph/9806029. 
[2] L. Babai, P. Frankl, and J. Simon. Complexity classes in communication complexity theory. 27th IEEE Symposium on Foundations of Computer Science, pp. 303-312, 1986.

[3] Z. Bar-Yossef, T. Jayram, R. Kumar, D. Sivakumar. An Information Statistics Approach to Data Stream and Communication Complexity. 43rd IEEE Symposium on Foundations of Computer Science, pp. 209$218,2002$.

[4] R. Bar-Yehuda, B. Chor, E. Kushilevitz, and A. Orlitsky. Privacy, Additional Information, and Communication. IEEE Transactions on Information Theory, vol. 39, pp. 1930-1943, 1993.

[5] D. Beaver. Perfect privacy for two party protocols. Technical Report TR-11-89, Harvard University, 1989.

[6] C.H. Bennett, E. Bernstein, G. Brassard, and U. Vazirani. Strengths and weaknesses of quantum computing. SIAM Journal on Computing, vol. 26, pp. 1510-1523, 1997. Also: quant-ph/9701001.

[7] M. Ben-Or, S. Goldwasser, and A. Wigderson. Completeness Theorems for Non-Cryptographic Fault-Tolerant Distributed Computation. 20th ACM Symposium on Theory of Computing, pp. 1-10, 1988.

[8] M. Boyer, G. Brassard, P. Hoyer, and A. Tapp. Tight bounds on quantum searching. 4th Workshop on Physics and Computation, pp. 36-43, 1996. Also: quant-ph/9605034.

[9] H. Buhrman, R. Cleve, and A. Wigderson. Quantum vs. classical communication and computation. 30th ACM Symposium on Theory of Computing, pp. 63-68, 1998. Also: quant-ph/9802040.

[10] V. Buzek and M. Hillery. Quantum Copying: Beyond the NoCloning Theorem. Phys. Rev. A, vol. 54, pp. 1844-1852, 1996. Also: quant-ph/9607018.

[11] A. Chakrabarti, Y. Shi, A. Wirth, and A.C.C. Yao. Informational Complexity and the Direct Sum Problem for Simultaneous Message Complexity. 42nd IEEE Symposium on Foundations of Computer Science, pp. 270-278, 2001.

[12] D. Chaum, C. Crepeau, and I. Damgard. Multiparty Unconditionally Secure Protocols. 20th ACM Symposium on Theory of Computing, pp. 11-19, 1988.

[13] B. Chor, M. Gereb-Graus, and E. Kushilevitz. On the Structure of the Privacy Hierarchy. Journal of Cryptology, vol. 7, pp. 53-60, 1994. 
[14] B. Chor and E. Kushilevitz. A zero-one law for Boolean privacy. SIAM Journal Discrete Math., vol. 4, pp. 36-47, 1991.

[15] R. Cleve, W. van Dam, M. Nielsen, and A. Tapp. Quantum Entanglement and the Communication Complexity of the Inner Product Function. 1st NASA Int. Conf. on Quantum Computing and Quantum Communications, 1998. Also: quant-ph/9708019.

[16] T.M. Cover and J.A. Thomas. Elements of Information Theory. Wiley Series in Telecommunications. John Wiley \& Sons, 1991.

[17] C. Crepeau, D. Gottesman, and A. Smith. Quantum Multi-party Computation, 34th ACM Symposium on Theory of Computing, pp. 643-652, 2002 .

[18] O. Goldreich, S. Micali, and A. Wigderson. How to Play Any Mental Game. 19th ACM Symposium on Theory of Computing, pp. 218-229, 1987.

[19] L.K. Grover. A fast quantum mechanical algorithm for database search. 28th ACM Symposium on Theory of Computing, pp. 212-219, 1996. Also: quant-ph/9605043.

[20] B. Kalyanasundaram and G. Schnitger. The Probabilistic Communication Complexity of Set Intersection. SIAM Journal Discrete Math., vol. 5, pp. 545-557, 1992.

[21] H. Klauck. Lower bounds for quantum communication complexity. 42nd IEEE Symposium on Foundations of Computer Science, pp. 288-297, 2001. Also: quant-ph/0106160.

[22] H. Klauck, A. Nayak, A. Ta-Shma, and D. Zuckerman. Interaction in Quantum Communication and the Complexity of Set Disjointness. 33rd ACM Symposium on Theory of Computing, pp. 124-133, 2001.

[23] E. Kushilevitz. Privacy and Communication Complexity. SIAM Journal Discrete Math., vol. 5, pp. 273-284, 1992.

[24] E. Kushilevitz and N. Nisan. Communication Complexity. Cambridge University Press, 1997.

[25] E. Kushilevitz, R. Ostrovsky, and A. Rosen. Characterizing Linear Size Circuits in Terms of Privacy. Journal of Computer and System Sciences, vol. 58, pp. 129-136, 1999.

[26] H.-K. Lo. Insecurity of Quantum Secure Computations. Phys. Rev. A, vol. 65, p. 1154, 1997. Also: quant-ph/9611031 
[27] H. Lo and H. Chau. Why quantum bit commitment and ideal quantum coin tossing are impossible. Physica D, vol. 120, pp. 177-187, 1998. Also: quant-ph/9711065

[28] I. Newman. Private vs. Common Random Bits in Communication Complexity. Information Processing Letters, vol. 39, pp. 67-71, 1991.

[29] M.A. Nielsen and I.L. Chuang. Quantum Computation and Quantum Information. Cambridge University Press, 2000.

[30] A.A. Razborov. Quantum Communication Complexity of Symmetric Predicates. Izvestiya: Math., vol. 67(1), pp. 145-159, 2003. Also: quant-ph/0204025.

[31] W.K. Wootters and W.H. Zurek. A single quantum cannot be cloned. Nature, vol. 299, pp. 802-803, 1982.

[32] A.C.-C. Yao. Protocols for Secure Computations. 23rd IEEE Symposium on Foundations of Computer Science, pp. 160-164, 1982.

[33] A.C.-C. Yao. Quantum circuit complexity. 34th IEEE Symposium on Foundations of Computer Science, pp. 352-361, 1993. 